\title{
Similarity measures for Fermatean fuzzy sets and its applications in group decision-making
}

\author{
Laxminarayan Sahoo ${ }^{\mathbf{a}^{*}}$
}

${ }^{a}$ Department of Computer and Information Science, Raiganj University, Raiganj-733134, India

\begin{tabular}{l}
\hline C H R O N I C L E \\
\hline Article history: \\
Received August 12, 2020 \\
Received in revised format: \\
July 17,2021 \\
Accepted November 252021 \\
Available online \\
November 25, 2021 \\
\hline Keywords: \\
Fermatean fuzzy set \\
Score function \\
Similarity measure \\
Multi-criteria decision making \\
Pattern recognition
\end{tabular}

\section{Introduction}

The theory of fuzzy set was established by Zadeh (Zadeh, 1965) in 1965 and it dealt with imprecision, vagueness in real life situations. In the year 1970, Bellman and Zadeh (1970) presented the notion of decision making problems entailing uncertainty. The concept of intuitionistic fuzzy sets (IFSs) was introduced by Atanassov ( Atanassov, 1986) in1986 by presenting the objective world from three aspects of support, opposition and neutrality, respectively and thus have been widely considered and applied by many researchers ( Fei et al., 2018; Zhang et al. 2018). Also, many researchers have given additional attention to interval valued fuzzy sets (IVFSs) (Turksen, 1986; Gorzalczany, 1987) interval valued intuitionistic fuzzy sets (IVIFSs) (Atanassov \& Gargov, 1987; Atanassov, 2012), which are all the generalization of the fuzzy set proposed by Zadeh (Zadeh, 1965) and applied them in diverse decision making problems. However, the fuzzy set takes only a membership function and the degree of non-membership function which is just a complement of the degree of membership function. There may be a situation where the sum of the membership function and non-membership function is greater than one. Thus orthopair fuzzy sets have been introduced in which the membership grades of an element $x \in X$ are pairs of values in the unit interval, $\langle\alpha(x), \beta(x)\rangle$, one of which indicates support of membership in the fuzzy set and other indicates support against membership in the fuzzy set. For example, Atanassov's classical intuitionistic fuzzy sets (Atanassov,1983; Atanassov, 1986; Atanassov et al., 2013) and Atanassov's second kind of intuitionistic fuzzy sets ( Atanassov, 2016). Yager (Yager, 2013; Yager, 2014) introduced another orthopair of fuzzy sets, known as Pythagorean fuzzy set (PFS), where the square sum of the support of membership and support against membership value is equal to or

* Corresponding author

E-mail address: 1xsahoo@gmail.com (L. Sahoo)

(C) 2022 by the authors; licensee Growing Science, Canada.

doi: $10.5267 / \mathrm{dsl} .2021 .11 .003$ 
less than one. Also, many researchers have paid more attention to interval valued Pythagorean fuzzy sets (IVPFSs) (Garg, 2017; Garg, 2018), which is the generalization of the Pythagorean fuzzy sets (PFSs) set proposed by Yager and applied them in several decision making problems. PFSs and IVPFSs have attracted the attention of many researchers within a very short period of time. There are several methods in the field of PFS to solve real-life multi-criteria, decision-making problems (Ye, 2009; Zhang et al., 2014; Zhang, 2016; Gou et al., 2016; Geng et al., 2017; Jing et al., 2017; Li et al., 2018). Several researchers have also proposed real-life applications under a Pythagorean fuzzy environment. For more details one may mention the works of Li et al. (Li et al., 2018), Zhou et al. (Zhou et al., 2018), Bolturk (Boltruk, 2018), Qin (Qin, 2018), Wan et al. (Wan et al., 2018), Lin et al. (Lin et al., 2018) and Chen (Chen, 2018). But, if orthopair fuzzy sets as $<0.9$, $0.6>$, where 0.9 is the support of the membership of certain criteria of a parameter and 0.6 is the support against membership then it does not follow the condition of IFS as well as PFS. However, the cubic sum of the support of membership and support against membership degrees is equal to or less than one. And in this situation Senapati and Yager (Senapati \& Yager, 2019; Senapati \& Yager, 2020) very recently introduced the Fermatean Fuzzy set (FFS). They also showed that FFSs have more uncertainty than IFSs and PFSs and are capable of handling higher levels of uncertainties (Bai, 2013) and solved MCDM problems.

A similarity measure is an important concept for controlling the degree of similarity between two objects in many fields, such as pattern recognition, medical diagnosis, personnel appointment etc. various types of similarity measures have been introduced (Pappis \& Karacapilidis, 1993; Chen, 1995; Li \& Cheng, 2002; Liang \& Shi, 2003 ; Hung \& Yang, 2004; Ye, 2011; Zhou et al., 2014; Liu et al., 2018 ). Among them, some similarity measures of intuitionistic fuzzy sets (IFSs) and Pythagorean fuzzy sets (PFSs) have been proposed. For example, Li and Cheng (2002) studied a similarity measure between IFSs and applied it to pattern recognition. Huang and Yang (2004) proposed the similarity measure between IFSs based on the Hausdorff distance and used it to calculate the degree of similarity between IFSs. Nguyen (2016) proposed a new knowledge-based similarity measure between IFSs and applied it to pattern recognition. Zhang (2016) introduced a novel approach based on similarity measures for Pythagorean fuzzy multiple criteria group decision making. Zhang et al. (2012) presents a type of score function on intuitionistic fuzzy sets with double parameters and its application to pattern recognition and medical diagnosis. Ejegwa (Ejegwa, 2020; Ejegwa, 2019) introduced distance and similarity measures of Pythagorean fuzzy sets. Ye et al. (2011) proposed a cosine similarity measure for IFSs (CIFS) and applied it to pattern recognition. Also, Ye (2013) presented the cosine similarity measure for IVIFSs (CIVIFS) and applied it to group decision-making problems. On the other hand Liu et al. (2018) studied Cosine Similarity Measure between Hybrid Intuitionistic Fuzzy Sets and Its Application in Medical Diagnosis. Very recently, Liu et al. (2019) introduced Distance measure for Fermatean fuzzy linguistic term sets based on linguistic scale function. But as per our knowledge and belief, no one studied the distance based similarity measure and cosine similarity measure based on score function between FFSs. Influenced by this, in this paper, we shall propose distance based similarity measure and the cosine similarity measure of FFSs to handle uncertain information. In addition, applying the proposed similarity measures, we have solved group decision-making problems which are very fascinating in the real-world. In this paper, we have introduced three newly improved score functions for ranking of Fermatean fuzzy sets. We have applied the proposed score function to calculate the similarity measure and applied it to solve pattern recognition problem viz. personnel appointment. Finally, a numerical example is given to illustrate the effectiveness of the proposed distance based and cosine similarity measures, which are also compared with the existing similarity measures.

The contributions of the present paper are the following. In section 2, some definitions and basic concepts related to Fermatean fuzzy sets are described. Section 3 gives the ranking of FFSs based on proposed score functions. In section 4, a similarity measure of Fermatean fuzzy sets has been presented. Section 5 gives the group decision making with similarity measures between FFSs. Numerical example is given in Section 6. Section 7 contains the conclusion of the paper with the future scope of research.

\section{Preliminaries}

In this section some basic definitions about Fermatean fuzzy sets (FFSs) are discussed. After that some score functions are proposed to implement the entire paper.

Definition 2.1. (Senapati \& Yager, 2019; Senapati \& Yager, 2020) Let $X$ be a Universal set. A Fermatean fuzzy set (FFS) is an object of the form $F=\left\{\left\langle x, \alpha_{F}(x), \beta_{F}(x)\right\rangle: x \in X\right\}$ where $\alpha_{F}(x): X \rightarrow[0,1]$ and $\beta_{F}(x): X \rightarrow[0,1]$ which

satisfies the relation $0 \leq\left(\alpha_{F}(x)\right)^{3}+\left(\beta_{F}(x)\right)^{3} \leq 1, \forall x \in X$. The number $\alpha_{F}(x)$ and $\beta_{F}(x)$ are the degree of membership and non-membership of the element $x \in X$ in the FFS $F$.

For any FFS $F$ and $x \in X$, the degree of indeterminacy is represented by $\pi_{F}(x)=\sqrt[3]{1-\left(\alpha_{F}(x)\right)^{3}-\left(\beta_{F}(x)\right)^{3}}$. It is to be noted that, for simplicity, we shall denote the object $F=\left\langle\alpha_{F}, \beta_{F}\right\rangle$ instead of $F=\left\{\left\langle x, \alpha_{F}(x), \beta_{F}(x)\right\rangle: x \in X\right\}$.

Definition 2.2. (Senapati \& Yager, 2019; Senapati \& Yager, 2020) Let $F_{1}=\left\langle\alpha_{F_{1}}, \beta_{F_{1}}\right\rangle$ and $F_{2}=\left\langle\alpha_{F_{2}}, \beta_{F_{2}}\right\rangle$ be two FFSs. Then the basic arithmetical operations of two Fermatean fuzzy sets $F_{1}$ and $F_{2}$ are defined as follows: 
(i) Addition: $F_{1} \oplus F_{2}=\left\langle\sqrt[3]{\left(\alpha_{F_{1}}\right)^{3}+\left(\alpha_{F_{2}}\right)^{3}-\left(\alpha_{F_{1}}\right)^{3}\left(\alpha_{F_{2}}\right)^{3}}, \beta_{F_{1}} \beta_{F_{2}}\right\rangle$

(ii) Multiplication: $F_{1} \otimes F_{2}=\left\langle\alpha_{F_{1}} \alpha_{F_{2}}, \sqrt[3]{\left(\beta_{F_{1}}\right)^{3}+\left(\beta_{F_{2}}\right)^{3}-\left(\beta_{F_{1}}\right)^{3}\left(\beta_{F_{2}}\right)^{3}}\right\rangle$

(iii) Scalar Multiplication: $\lambda \odot F=\left\langle\sqrt[3]{1-\left(1-\left(\alpha_{F}\right)^{3}\right)^{\lambda}},\left(\beta_{F}\right)^{\lambda}\right\rangle$ provided $\lambda>0$

(iv) Exponent: $F^{\lambda}=\left\langle\left(\alpha_{F}\right)^{\lambda}, \sqrt[3]{1-\left(1-\left(\beta_{F}\right)^{3}\right)^{\lambda}}\right\rangle$

Definition 2.3. (Senapati \& Yager, 2019; Senapati \& Yager, 2020) Let $F_{1}=\left\langle\alpha_{F_{1}}, \beta_{F_{1}}\right\rangle$ and $F_{2}=\left\langle\alpha_{F_{2}}, \beta_{F_{2}}\right\rangle$ be two FFSs. Then their set operations are defined as follows:

(i) Union: $F_{1} \cup F_{2}=\left\langle\max \left(\alpha_{F_{1}} \alpha_{F_{2}}\right), \min \left(\beta_{F_{1}} \beta_{F_{2}}\right)\right\rangle$

(ii) Intersection: $F_{1} \cap F_{2}=\left\langle\min \left(\alpha_{F_{1}} \alpha_{F_{2}}\right), \max \left(\beta_{F_{1}} \beta_{F_{2}}\right)\right\rangle$

(iii) Compliment: $\left(F_{1}\right)^{\prime}=\left\langle\beta_{F_{1}}, \alpha_{F_{1}}\right\rangle$

Definition 2. 4. (Senapati \& Yager, 2019; Senapati \& Yager, 2020) Let $F_{1}=\left\langle\alpha_{F_{1}}, \beta_{F_{1}}\right\rangle, F_{2}=\left\langle\alpha_{F_{2}}, \beta_{F_{2}}\right\rangle$ and $F_{2}=\left\langle\alpha_{F_{3}}, \beta_{F_{3}}\right\rangle$ are three FFSs. Then the following properties hold:

(i) $F_{1} \cup F_{2}=F_{2} \cup F_{1}$

(ii) $F_{1} \cap F_{2}=F_{2} \cap F_{3}$

(iii) $F_{1} \cup\left(F_{2} \cup F_{3}\right)=\left(F_{1} \cup F_{2}\right) \cup F_{3}$

(iv) $F_{1} \cap\left(F_{2} \cap F_{3}\right)=\left(F_{1} \cap F_{2}\right) \cap F_{3}$

(v) $\lambda \cup\left(F_{1} \cup F_{2}\right)=\lambda F_{1} \cup \lambda F_{2}$

(vi) $\left(F_{1} \cup F_{2}\right)^{\lambda}=F_{1}^{\lambda} \cup F_{2}^{\lambda}$

Definition 2. 5. (Senapati \& Yager, 2019; Senapati \& Yager, 2020) Let $F=\left\langle\alpha_{F}, \beta_{F}\right\rangle$ be any FFS then score function of $F$ denoted by $\psi(F)$ and is defined by $\psi(F)=\left(\alpha_{F}^{3}-\beta_{F}^{3}\right)$. Here, the score function $\psi(F) \in[-1,1]$.

Definition 2.6. (Senapati \& Yager, 2019; Senapati \& Yager, 2020) Let $F=\left\langle\alpha_{F}, \beta_{F}\right\rangle$ be any FFS then accuracy function of $F$ denoted by $H(F)$ and is defined by $H(F)=\alpha_{F}^{3}+\beta_{F}^{3}$.

\section{Ranking of Fermatean fuzzy sets}

For the purpose of ranking, we have proposed some score functions $\psi(F), \psi(F) \in[0,1]$ which are as follows:

(i) $\left(\right.$ Type 1) $\psi_{1}(F)=\frac{1-\beta_{F}{ }^{3}}{2-\alpha_{F}{ }^{3}-\beta_{F}{ }^{3}}$

(iii) $($ Type 2$) \psi_{2}(F)=\frac{1}{3}\left(1+2 \alpha_{F}^{3}-\beta_{F}^{3}\right)$

(ii) $\left(\right.$ Type 3) $\psi_{3}(F)=\frac{1}{2}\left(1+\alpha_{F}^{3}-\beta_{F}^{3}\right)$

Property 3.1. Let $F=\left\langle\alpha_{F}, \beta_{F}\right\rangle$ be any FFS then $\psi_{1}(F) \in[0,1], \psi_{2}(F) \in[0,1]$ and $\psi_{3}(F) \in[0,1]$. 
Proof: For any FFS $\alpha_{F}^{3} \geq 0$ and $\beta_{F}^{3} \leq 1 \Rightarrow 1-\beta_{F}^{3} \geq 0$. Hence, $1+\alpha_{F}^{3}-\beta_{F}^{3} \geq 0$ and obviously $\psi_{3}(F) \geq 0$. Again, $\alpha_{F}^{3}+\beta_{F}^{3} \leq 1 \Rightarrow 1+\alpha_{F}^{3}+\beta_{F}^{3} \leq 2 \Rightarrow 1+\alpha_{F}^{3}-\beta_{F}^{3} \leq 2$ as $\beta_{F}^{3} \geq 0$. So $\frac{1+\alpha_{F}^{3}-\beta_{F}^{3}}{2} \leq 1$ and $\psi_{3}(F) \leq 1$.

Hence for Type 3 score function $\psi_{3}(F) \in[0,1]$. In similar manner one can prove that $\psi_{1}(F) \in[0,1]$ and $\psi_{2}(F) \in[0,1]$. Particularly, if $F=\langle 0,1\rangle$, then $\psi_{1}(F)=0, \psi_{2}(F)=0$ and $\psi_{3}(F)=0$. Again if $F=\langle 1,0\rangle$, then $\psi_{1}(F)=1, \psi_{2}(F)=1$ and $\psi_{3}(F)=1$.

Example 3.1. (Senapati \& Yager, 2019; Senapati \& Yager, 2020) If $F_{1}=\langle 0.93,0.60\rangle$ and $F_{2}=\langle 0.85,0.75\rangle$ then we have the following:

For Type 1. $\psi_{1}\left(F_{1}\right)=0.8003$ and $\psi_{1}\left(F_{2}\right)=0.5997$. Therefore, $\psi_{1}\left(F_{1}\right)>\psi_{1}\left(F_{2}\right) \Rightarrow F_{1}>F_{2}$.

For Type 2. $\psi_{2}\left(F_{1}\right)=0.7976$ and $\psi_{2}\left(F_{2}\right)=0.6021$. Therefore, $\psi_{2}\left(F_{1}\right)>\psi_{2}\left(F_{2}\right) \Rightarrow F_{1}>F_{2}$.

For Type 3. $\psi_{3}\left(F_{1}\right)=0.9842$ and $\psi_{3}\left(F_{2}\right)=0.5961$. Therefore, $\psi_{3}\left(F_{1}\right)>\psi_{3}\left(F_{2}\right) \Rightarrow F_{1}>F_{2}$.

So we claim that our proposed score functions are justified as all score functions gives the same results similar to Senapati and Yager (Senapati \& Yager, 2019; Senapati \& Yager, 2020).

Again if $F=\left\langle\alpha_{F}, \alpha_{F}\right\rangle$ then $\psi_{2}(F)=\psi_{3}(F)=0.5$ whereas $\psi_{3}(F)$ may not be 0.5 always.

It is either greater than equal to 0.5 or less than 0.5 . If we consider $F_{1}=\left\langle\frac{\sqrt[3]{4}}{2}, \frac{\sqrt[3]{4}}{2}\right\rangle$ and $F_{2}=\langle 0.5,0.5\rangle$ then $\psi_{1}\left(F_{1}\right)=\psi_{2}\left(F_{1}\right)=\psi_{3}\left(F_{1}\right)=0.5$ and $\psi_{1}\left(F_{2}\right)=\psi_{2}\left(F_{2}\right)=\psi_{3}\left(F_{2}\right)=0.5$. So score values are same although $F_{1}=\left\langle\frac{\sqrt[3]{4}}{2}, \frac{\sqrt[3]{4}}{2}\right\rangle$ and $F_{2}=\langle 0.5,0.5\rangle$ are different. Hence, for ranking purpose another measure be needed and then accuracy function may be defined.

Definition 3.1. Let $F_{1}=\left\langle\alpha_{F_{1}}, \beta_{F_{1}}\right\rangle$ and $F_{2}=\left\langle\alpha_{F_{2}}, \beta_{F_{2}}\right\rangle$ be two FFSs. Then ranking or order relations of $F_{1}$ and $F_{2}$ are defined as follows:

(i) $F_{1} \succ_{\max } F_{2}$ iff either $\left(S\left(F_{1}\right)>S\left(F_{2}\right)\right)$ or $\left(S\left(F_{1}\right)=S\left(F_{2}\right)\right.$ and $\left.H\left(F_{1}\right)>H\left(F_{2}\right)\right)$

(ii) $F_{1} \prec_{\min } F_{2}$ iff either $\left(S\left(F_{1}\right)<S\left(F_{2}\right)\right)$ or $\left(S\left(F_{1}\right)=S\left(F_{2}\right)\right.$ and $\left.H\left(F_{1}\right)<H\left(F_{2}\right)\right)$

(iii) $F_{1} \simeq_{\text {equal }} F_{2}$ iff $S\left(F_{1}\right)=S\left(F_{2}\right)$ and $H\left(F_{2}\right)=H\left(F_{2}\right)$

From above it is noticed that if $F_{1}=\left\langle\frac{\sqrt[3]{4}}{2}, \frac{\sqrt[3]{4}}{2}\right\rangle$ and $F_{2}=\langle 0.5,0.5\rangle$ then $S\left(F_{1}\right)=0.5$ and $S\left(F_{2}\right)=0.5$ for all the three types of score function but $H\left(F_{1}\right)=1.0$ and $H\left(F_{2}\right)=0.25$ hence $F_{1} \succ_{\max } F_{2}$.

\section{Similarity measures for Fermatean fuzzy sets}

The similarity measure can measure the similarity degree between two different alternatives. In this section we have proposed some score based similarity measures with Fermatean fuzzy sets using the concept of distance metric and some properties are also presented.

Definition 4.1. Let $X$ be a Universal set and $F_{i}=\left\{\left\langle x, \alpha_{i_{F}}(x), \beta_{i_{F}}(x)\right\rangle: x \in X\right\}, i=1,2,3$ where $\alpha_{i F}(x): X \rightarrow[0,1]$ and $\beta_{i F}(x): X \rightarrow[0,1]$ are three FFSs. The similarity measure $S$ between $F_{1}$ and $F_{2}$ is a mapping $S: X \times X \rightarrow[0,1]$ satisfies the following conditions:

(ii) $\quad S\left(F_{1}, F_{2}\right)=1 \Leftrightarrow F_{1}=F_{2}, F_{1}, F_{2} \in X$

(iii) $\quad S\left(F_{1}, F_{2}\right)=S\left(F_{2}, F_{1}\right), \forall F_{1}, F_{2} \in X$

(iv) $\quad S\left(F_{1}, F_{2}\right) \leq S\left(F_{1}, F_{3}\right)+S\left(F_{3}, F_{2}\right), \forall F_{1}, F_{2}, F_{3} \in X$

The condition (i) expresses that $S$ is bounded-ness in $F_{1}, F_{2}$. Condition (iii) gives the fact that $S$ is symmetric in $F_{1}, F_{2}$. The inequality (iv) is generally called the triangle inequality.

Let us assume that there are two PFSs $A$ and $P$ in $X=\left\{x_{1}, x_{2}, \ldots, x_{n}\right\}$. Also let $\psi$ be the score function. Then the similarity measure between $A$ and $P$ as follows: 


$$
\begin{aligned}
& S(A, P)=1-\left(\frac{1}{2 n} \sum_{i=1}^{n}\left(\left|\alpha_{A i}-\alpha_{P i}\right|^{3}+\left|\beta_{A i}-\beta_{P i}\right|^{3}+\left|\pi_{A i}-\pi_{P i}\right|^{3}\right)\right)^{\frac{1}{3}} \\
& S(A, P)=1-\frac{1}{2 n} \sum_{i=1}^{n}\left(\left|\alpha_{A i}-\alpha_{P i}\right|+\left|\beta_{A i}-\beta_{P i}\right|+\left|\pi_{A i}-\pi_{P i}\right|\right) \\
& S(A, P)=1-\frac{1}{2 n} \sum_{i=1}^{n}\left(\left|\alpha_{A i}{ }^{3}-\alpha_{P i}{ }^{3}\right|+\left|\beta_{A i}{ }^{3}-\beta_{P i}{ }^{3}\right|+\left|\pi_{A i}{ }^{3}-\pi_{P i}{ }^{3}\right|\right) \\
& S(A, P)=1-\left(\frac{1}{2 n} \sum_{i=1}^{n}\left(\mid \psi\left(A_{i}\right)-\psi\left(P_{i}\right)^{3}\right)\right)^{\frac{1}{3}} \\
& S(A, P)=1-\frac{1}{2 n} \sum_{i=1}^{n}\left(\left|\psi\left(A_{i}\right)-\psi\left(P_{i}\right)\right|\right) \\
& S(A, P)=1-\frac{1}{2 n} \sum_{i=1}^{n}\left(\left|\left(\psi\left(A_{i}\right)\right)^{3}-\left(\psi\left(P_{i}\right)\right)^{3}\right|\right) \\
& S(A, P)=1-\frac{1}{2 n} \sum_{i=1}^{n}\left(\frac{1}{2^{i}} \frac{\left|\psi\left(A_{i}\right)-\psi\left(P_{i}\right)\right|}{1+\left|\psi\left(A_{i}\right)-\psi\left(P_{i}\right)\right|}\right) \\
& S(A, P)=1-\frac{1}{2 n} \max _{i}\left(\left|\psi\left(A_{i}\right)-\psi\left(P_{i}\right)\right|\right)
\end{aligned}
$$

Now, we have also defined score based Cosine similarity measure as follows:

$$
\operatorname{CosFFS}(A, P)=\frac{1}{n} \sum_{i=1}^{n}\left(\frac{\psi\left(A_{i}\right) \psi\left(P_{i}\right)+\pi_{A i}^{*} \pi_{P i}}{\sqrt{\psi^{2}\left(A_{i}\right)+\pi_{A i}^{2}} \sqrt{\psi^{2}\left(P_{i}\right)+\pi_{P i}^{2}}}\right)
$$

Theorem 4.1. The cosine similarity measures between two Fermatean fuzzy sets $A$ and $P$ satisfies the following properties:
(a) $0 \leq \operatorname{CosFFS}(A, P) \leq 1$
(b) $\operatorname{CosFFS}(A, P)=\operatorname{CosFFS}(A, P)$
(c) $\operatorname{CosFFS}(A, P)=1$, if $A=P$

Proof: It is obvious that the property (a) is true according to the cosine value in $[0,1]$

(b) Multiplication of real numbers satisfies the commutative law, hence if the positions of $A$ and $P$ are interchanged then the formula of cosine measure gives the same results.

(c) Let $A_{i}=<\alpha_{A i}, \beta_{A i}>$ and $P=<\alpha_{P i}, \beta_{P i}>$. Now, if $A_{i}=P_{i}$ then $\alpha_{A i}=\alpha_{P i}$ and $\beta_{A i}=\beta_{P i}$. So, $\psi\left(A_{i}\right)=\psi\left(P_{i}\right)$ and $\pi_{A i}=\pi_{P_{i}}$ for all $i=1,2, \ldots, n$.

Now, $\operatorname{CosFFS}(A, P)=\frac{1}{n} \sum_{i=1}^{n}\left(\frac{\psi\left(A_{i}\right) \psi\left(P_{i}\right)+\pi_{A i} * \pi_{P i}}{\sqrt{\psi^{2}\left(A_{i}\right)+\pi_{A i}^{2}} \sqrt{\psi^{2}\left(P_{i}\right)+\pi_{P i}^{2}}}\right)=\frac{1}{n} \sum_{i=1}^{n}\left(\frac{\psi^{2}\left(A_{i}\right) \psi\left(P_{i}\right)+\pi^{2}{ }_{A i}}{\sqrt{\psi^{2}\left(A_{i}\right)+\pi_{A i}^{2}} \sqrt{\psi^{2}\left(A_{i}\right)+\pi_{A i}^{2}}}\right)$

$$
=\frac{1}{n} \sum_{i=1}^{n}\left(\frac{\psi^{2}\left(A_{i}\right) \psi\left(P_{i}\right)+\pi_{A i}^{2}}{\sqrt{\psi^{2}\left(A_{i}\right)+\pi_{A i}^{2}} \sqrt{\psi^{2}\left(A_{i}\right)+\pi_{A i}^{2}}}\right)=\frac{1}{n} \sum_{i=1}^{n}\left(\frac{\psi^{2}\left(A_{i}\right) \psi\left(P_{i}\right)+\pi^{2}{ }_{A i}}{\psi^{2}\left(A_{i}\right)+\pi_{A i}^{2}}\right)=\frac{1}{n} \sum_{i=1}^{n}(1)=\frac{1}{n} n=1 .
$$

\section{Group Decision-Making with Similarity Measure between Fermatean fuzzy sets}

In this section, we shall apply different similarity measure between Fermatean fuzzy sets in personnel appointments problem. Here, we need several experts to evaluate properly the candidates/applicants of a particular competitive examination or personal interview for employment. The panel expert usually provides his/her preferences for candidates' qualification as well as positions in terms of Fermatean fuzzy sets.

Let us assume that a Company has organized an aptitude test for the selection of employees. 
Assume that $A_{1}$ is a set of qualifications $P$ is a set of positions and $A$ is a set of applicants. It is to be noted that for this organization (Company), all positions do not have same qualifications and it may varies. Let $X=\left\{x_{1}, x_{2}, \ldots, x_{n}\right\}$, $P=\left\{P_{1}, P_{2}, \ldots, P_{n}\right\}$ and $A=\left\{A_{1}, A_{2}, \ldots, A_{m}\right\}$ be finite set of qualifications, positions and applicants respectively.

As per our considerations $\quad P_{j}\left(x_{i}\right)=<p_{j_{\alpha i}}, p_{j \beta i}>$ and $p_{j_{\alpha i}}^{3}+p_{j_{\beta i}}^{3} \leq 1, i=1,2, \ldots, n ; j=1,2, \ldots, n \quad$ and $A_{j}\left(x_{i}\right)=<a_{j_{\alpha i}}, a_{j \beta}>$ and $a_{j_{\alpha i}}^{3}+a_{j_{\beta i}}^{3} \leq 1, i=1,2, \ldots, n ; j=1,2, \ldots, m$ are Fermatean fuzzy sets. For finding the decision experts will have to calculate the similarity measures between $A$ and $P$ i.e., $S(A, P)$ using different similarity measures and his/her opinion an applicant $A_{j}$ is appropriate for position $P_{i}$ if $S\left(a_{j}, p_{i}\right)$ is greatest. In tabular form, this problem can be represented as follows:

Table 1

Applicants vs. Qualifications

\begin{tabular}{cccccc} 
Applicants & $x_{1}$ & $x_{2}$ & $x_{3}$ & $\cdots$ & $x_{n}$ \\
\hline$A_{1}$ & $<a_{1 \alpha 1}, a_{1 \beta 1}>$ & $<a_{1 \alpha 2}, a_{1 \beta 2}>$ & $<a_{1 \alpha 3}, a_{1 \beta 3}>$ & $\ldots$ & $<a_{1 \alpha n}, a_{1 \beta n}>$ \\
$A_{2}$ & $<a_{2 \alpha 1}, a_{2 \beta 1}>$ & $<a_{2 \alpha 2}, a_{2 \beta 2}>$ & $<a_{2 \alpha 3}, a_{2 \beta 3}>$ & $\ldots$ & $<a_{2 \alpha n}, a_{2 \beta n}>$ \\
$\vdots$ & $\vdots$ & $\vdots$ & $\vdots$ & $\vdots$ & $\vdots$ \\
$A_{m}$ & $<a_{m \alpha 1}, a_{m \beta 1}>$ & $<a_{m \alpha 2}, a_{m \beta 2}>$ & $<a_{m \alpha 3}, a_{m \beta 3}>$ & $\ldots$ & $<a_{m \alpha n}, a_{m \beta n}>$
\end{tabular}

Table 2

Qualification vs. positions

\begin{tabular}{cccccc} 
& $P_{1}$ & $P_{2}$ & $P_{3}$ & $\ldots$ & $P_{n}$ \\
\hline$x_{1}$ & $<p_{1 \alpha 1}, p_{1 \beta 1}>$ & $<p_{1 \alpha 2}, p_{1 \beta 2}>$ & $<p_{1 \alpha 3}, p_{1 \beta 3}>$ & $\ldots$ & $<p_{1 \alpha n}, p_{1 \beta n}>$ \\
$x_{2}$ & $<p_{2 \alpha 1}, p_{2 \beta 1}>$ & $<p_{2 \alpha 2}, p_{2 \beta 2}>$ & $<p_{2 \alpha 3}, p_{2 \beta 3}>$ & $\ldots$ & $<p_{2 \alpha n}, p_{2 \beta n}>$ \\
$x_{3}$ & $<p_{3 \alpha 1}, p_{3 \beta 1}>$ & $<p_{3 \alpha 2}, p_{3 \beta 2}>$ & $<p_{3 \alpha 3}, p_{3 \beta 3}>$ & $\ldots$ & $<p_{3 \alpha n}, p_{3 \beta n}>$ \\
$\vdots$ & $\vdots$ & $\vdots$ & $\vdots$ & $\vdots$ & $\vdots$ \\
$x_{n}$ & $<a_{n \alpha 1}, a_{n \beta 1}>$ & $<a_{n \alpha 2}, a_{n \beta 2}>$ & $<a_{n \alpha 3}, a_{n \beta 3}>$ & $\ldots$ & $<a_{n \alpha n}, a_{n \beta n}>$
\end{tabular}

Table 3

Similarity measure of Applicants vs. Positions using $S(A, P)$

\begin{tabular}{|c|c|c|c|c|c|}
\hline$S(A, P)$ & $P_{1}$ & $P_{2}$ & $P_{3}$ & $\ldots$ & $P_{n}$ \\
\hline$A_{1}$ & $S\left(a_{1}, p_{1}\right)$ & $S\left(a_{1}, p_{2}\right)$ & $S\left(a_{1}, p_{3}\right)$ & $\cdots$ & $S\left(a_{1}, p_{n}\right)$ \\
\hline$A_{2}$ & $S\left(a_{2}, p_{1}\right)$ & $S\left(a_{2}, p_{2}\right)$ & $S\left(a_{2}, p_{3}\right)$ & $\ldots$ & $S\left(a_{2}, p_{n}\right)$ \\
\hline$\vdots$ & $\vdots$ & $\vdots$ & $\vdots$ & $\vdots$ & $\vdots$ \\
\hline$A_{m}$ & $S\left(a_{m}, p_{1}\right)$ & $S\left(a_{m}, p_{2}\right)$ & $S\left(a_{m}, p_{3}\right)$ & $\ldots$ & $S\left(a_{m}, p_{n}\right)$ \\
\hline
\end{tabular}

After construction of Table 5.3 decision makers have to take decision as per their requirements. They should take horizontal decision if the organization can requites all the applicants. Also decision makers should take vertical decision if the organization has limited fund/resources to pay salary. It is to be noted that vertical decision is more competitive compare to horizontal decision.

\section{Numerical example and discussion}

In this section, we have taken a numerical example to discuss the proposed approach.

Example 6.1.

Let $A=\left\{A_{1}, A_{2}, A_{3}, A_{4}\right\}$ be the set of applicants vying for positions, $P=\left\{P_{1}, P_{2}, P_{3}, P_{4}, P_{5}\right\}$ be the set of positions and $X=\{$ Honesty,Team Spirit, Hardworking, Transparency,Academic Fitness $\}$ be the set of qualifications expected by the applicants. Assume that the grades of the interview are captured by the decision makers in Fermatean fuzzy orthopair as presented below: 
Table 4

Applicants vs. Qualifications

\begin{tabular}{cccccc} 
Applicants & Honesty & Team Spirit & Hardworking & Transparency & Academic Fitness \\
\hline$A_{1}$ & $<0.5,0.1>$ & $<0.6,0.1>$ & $<0.7,0.2>$ & $<0.8,0.1>$ & $<0.5,0.2>$ \\
$A_{2}$ & $<0.8,0.1>$ & $<0.6,0.2>$ & $<0.7,0.1>$ & $<0.6,0.2>$ & $<0.4,0.5>$ \\
$A_{3}$ & $<0.5,0.2>$ & $<0.6,0.1>$ & $<0.5,0.1>$ & $<0.5,0.2>$ & $<0.8,0.1>$ \\
$A_{4}$ & $<0.7,0.1>$ & $<0.5,0.3>$ & $<0.8,0.1>$ & $<0.6,0.2>$ & $<0.7,0.1>$ \\
\hline
\end{tabular}

Let us assumed that Table 5 gives the stipulated standing qualifications by the expert panel, for each of the positions as below:

Table 5

Qualification vs. positions

\begin{tabular}{cccccc} 
& $P_{1}$ & $P_{2}$ & $P_{3}$ & $P_{4}$ & $P_{5}$ \\
\hline Honesty & $<0.7,0.2>$ & $<0.7,0.3>$ & $<0.6,0.2>$ & $<0.8,0.1>$ & $<0.6,0.3>$ \\
Team Spirit & $<0.8,0.1>$ & $<0.7,0.2>$ & $<0.8,0.0>$ & $<0.6,0.2>$ & $<0.8,0.1>$ \\
Hardworking & $<0.8,0.2>$ & $<0.8,0.1>$ & $<0.8,0.1>$ & $<0.7,0.2>$ & $<0.8,0.1>$ \\
Transparency & $<0.7,0.2>$ & $<0.7,0.1>$ & $<0.9,0.0>$ & $<0.8,0.1>$ & $<0.7,0.1>$ \\
Academic Fitness & $<0.9,0.1>$ & $<0.9,0.0>$ & $<0.6,0.3>$ & $<0.7,0.2>$ & $<0.5,0.3>$
\end{tabular}

The example is numerically same with the example solved by Ejegwa (Ejegwa, 2019) but descriptively different. Now using our proposed similarity measures we have constructed the following tables as below:

\section{Table 6}

Similarity measure of Applicants vs. Positions using (1)

\begin{tabular}{cccccc} 
Applicants & $P_{1}$ & $P_{2}$ & $P_{3}$ & $P_{4}$ & $P_{5}$ \\
\hline$A_{1}$ & 0.7571 & 0.7512 & 0.8248 & 0.8240 & 0.8306 \\
$A_{2}$ & 0.7267 & 0.6969 & 0.7468 & 0.8091 & 0.8043 \\
$A_{3}$ & 0.7914 & 0.7957 & 0.7206 & 0.7807 & 0.7584 \\
$A_{4}$ & 0.7926 & 0.8110 & 0.7397 & 0.8382 & 0.7914
\end{tabular}

Table 7

Similarity measure of Applicants vs. Positions using (2)

\begin{tabular}{cccccc} 
Applicants & $P_{1}$ & $P_{2}$ & $P_{3}$ & $P_{4}$ & $P_{5}$ \\
\hline$A_{1}$ & 0.8006 & 0.7878 & 0.8468 & 0.9140 & 0.8752 \\
$A_{3}$ & 0.7663 & 0.7759 & 0.7661 & 0.8798 & 0.8297 \\
$A_{3}$ & 0.8276 & 0.8154 & 0.7531 & 0.8094 & 0.7909 \\
$A_{4}$ & 0.8558 & 0.8636 & 0.7876 & 0.8776 & 0.8270
\end{tabular}

Table 8

Similarity measure of Applicants vs. Positions using (3)

\begin{tabular}{cccccc} 
Applicants & $P_{1}$ & $P_{2}$ & $P_{3}$ & $P_{4}$ & $P_{5}$ \\
\hline$A_{1}$ & 0.7074 & 0.7360 & 0.8220 & 0.8776 & 0.8460 \\
$A_{2}$ & 0.7134 & 0.7486 & 0.7148 & 0.8836 & 0.8028 \\
$A_{3}$ & 0.7314 & 0.7614 & 0.6652 & 0.7650 & 0.7204 \\
$A_{4}$ & 0.8172 & 0.8486 & 0.7692 & 0.8536 & 0.8282
\end{tabular}

Table 9

Similarity measure of Applicants vs. Positions using (4) and Type-1 score function

\begin{tabular}{cccccc} 
Applicants & $P_{1}$ & $P_{2}$ & $P_{3}$ & $P_{4}$ & $P_{5}$ \\
\hline$A_{1}$ & 0.8761 & 0.8791 & 0.9310 & 0.9329 & 0.9409 \\
$A_{2}$ & 0.8556 & 0.8576 & 0.8843 & 0.9322 & 0.9289 \\
$A_{3}$ & 0.9148 & 0.9211 & 0.8692 & 0.9147 & 0.9091 \\
$A_{4}$ & 0.9023 & 0.9125 & 0.8851 & 0.9400 & 0.9280
\end{tabular}

Table 10 
174

Similarity measure of Applicants vs. Positions using (4) and Type-2 score function

\begin{tabular}{cccccc} 
Applicants & $P_{1}$ & $P_{2}$ & $P_{3}$ & $P_{4}$ & $P_{5}$ \\
\hline$A_{1}$ & 0.8000 & 0.8063 & 0.8918 & 0.8735 & 0.8975 \\
$A_{2}$ & 0.7677 & 0.7720 & 0.8175 & 0.8754 & 0.8755 \\
$A_{3}$ & 0.8503 & 0.8613 & 0.8742 & 0.8416 & 0.8323 \\
$A_{4}$ & 0.8459 & 0.8714 & 0.8168 & 0.8961 & 0.8660
\end{tabular}

Table 11

Similarity measure of Applicants vs. Positions using (4) and Type-3 score function

\begin{tabular}{cccccc} 
Applicants & $P_{1}$ & $P_{2}$ & $P_{3}$ & $P_{4}$ & $P_{5}$ \\
\hline$A_{1}$ & 0.8493 & 0.8541 & 0.9187 & 0.9051 & 0.9230 \\
$A_{2}$ & 0.8121 & 0.8146 & 0.8604 & 0.8960 & 0.9032 \\
$A_{3}$ & 0.8882 & 0.8963 & 0.8368 & 0.8803 & 0.8724 \\
$A_{4}$ & 0.8824 & 0.9027 & 0.8602 & 0.9210 & 0.8956
\end{tabular}

Table 12

Similarity measure of Applicants vs. Positions using (5) and Type-1 score function

\begin{tabular}{cccccc} 
Applicants & $P_{1}$ & $P_{2}$ & $P_{3}$ & $P_{4}$ & $P_{5}$ \\
\hline$A_{1}$ & 0.9426 & 0.9501 & 0.9655 & 0.9789 & 0.9724 \\
$A_{2}$ & 0.9403 & 0.9465 & 0.9406 & 0.9767 & 0.9612 \\
$A_{3}$ & 0.9496 & 0.9567 & 0.9349 & 0.9579 & 0.9510 \\
$A_{4}$ & 0.9625 & 0.9690 & 0.9532 & 0.9714 & 0.9684
\end{tabular}

Table 13

Similarity measure of Applicants vs. Positions using (5) and Type-2 score function

\begin{tabular}{cccccc} 
Applicants & $P_{1}$ & $P_{2}$ & $P_{3}$ & $P_{4}$ & $P_{5}$ \\
\hline$A_{1}$ & 0.9027 & 0.9148 & 0.9430 & 0.9594 & 0.9517 \\
$A_{2}$ & 0.9006 & 0.9109 & 0.9009 & 0.9573 & 0.9321 \\
$A_{3}$ & 0.9112 & 0.9228 & 0.8872 & 0.9219 & 0.9076 \\
$A_{4}$ & 0.9387 & 0.9495 & 0.9208 & 0.9503 & 0.9399
\end{tabular}

Table 14

Similarity measure of Applicants vs. Positions using (5) and Type-3 score function

\begin{tabular}{cccccc} 
Applicants & $P_{1}$ & $P_{2}$ & $P_{3}$ & $P_{4}$ & $P_{5}$ \\
\hline$A_{1}$ & 0.9269 & 0.9366 & 0.9577 & 0.9694 & 0.9638 \\
$A_{2}$ & 0.9222 & 0.9293 & 0.9227 & 0.9647 & 0.9457 \\
$A_{3}$ & 0.9336 & 0.9426 & 0.9146 & 0.9409 & 0.9304 \\
$A_{4}$ & 0.9530 & 0.9608 & 0.9389 & 0.9618 & 0.9528
\end{tabular}

Table 15

Similarity measure of Applicants vs. Positions using (6) and Type-1 score function

\begin{tabular}{cccccc} 
Applicants & $P_{1}$ & $P_{2}$ & $P_{3}$ & $P_{4}$ & $P_{5}$ \\
\hline$A_{1}$ & 0.9301 & 0.9391 & 0.9560 & 0.9779 & 0.9681 \\
$A_{2}$ & 0.9287 & 0.9363 & 0.9288 & 0.9764 & 0.9576 \\
$A_{3}$ & 0.9404 & 0.9490 & 0.9226 & 0.9540 & 0.9474 \\
$A_{4}$ & 0.9528 & 0.9609 & 0.9435 & 0.9672 & 0.9674
\end{tabular}

Table 16 
Similarity measure of Applicants vs. Positions using (6) and Type-2 score function

\begin{tabular}{cccccc} 
Applicants & $P_{1}$ & $P_{2}$ & $P_{3}$ & $P_{4}$ & $P_{5}$ \\
\hline$A_{1}$ & 0.8961 & 0.9096 & 0.9360 & 0.9660 & 0.9507 \\
$A_{2}$ & 0.8962 & 0.9082 & 0.8962 & 0.9660 & 0.9366 \\
$A_{3}$ & 0.9120 & 0.9251 & 0.8848 & 0.9293 & 0.9191 \\
$A_{4}$ & 0.9316 & 0.9442 & 0.9173 & 0.9498 & 0.9507
\end{tabular}

Table 17

Similarity measure of Applicants vs. Positions using (6) and Type-3 score function

\begin{tabular}{cccccc} 
Applicants & $P_{1}$ & $P_{2}$ & $P_{3}$ & $P_{4}$ & $P_{5}$ \\
\hline$A_{1}$ & 0.8941 & 0.9085 & 0.9365 & 0.9619 & 0.9489 \\
$A_{2}$ & 0.8914 & 0.9023 & 0.8916 & 0.9591 & 0.9295 \\
$A_{3}$ & 0.9085 & 0.9220 & 0.8799 & 0.9228 & 0.9110 \\
$A_{4}$ & 0.9304 & 0.9425 & 0.9134 & 0.9467 & 0.9424
\end{tabular}

Table 18

Similarity measure of Applicants vs. Positions using (7) and Type-1 score function

\begin{tabular}{cccccc} 
Applicants & $P_{1}$ & $P_{2}$ & $P_{3}$ & $P_{4}$ & $P_{5}$ \\
\hline$A_{1}$ & 0.9924 & 0.9942 & 0.9947 & 0.9937 & 0.9952 \\
$A_{2}$ & 0.9924 & 0.9937 & 0.9902 & 0.9990 & 0.9910 \\
$A_{3}$ & 0.9920 & 0.9937 & 0.9930 & 0.9920 & 0.9941 \\
$A_{4}$ & 0.9960 & 0.9972 & 0.9934 & 0.9946 & 0.9940
\end{tabular}

Table 19

Similarity measure of Applicants vs. Positions using (7) and Type-2 score function

\begin{tabular}{cccccc} 
Applicants & $P_{1}$ & $P_{2}$ & $P_{3}$ & $P_{4}$ & $P_{5}$ \\
\hline$A_{1}$ & 0.9868 & 0.9893 & 0.9909 & 0.9893 & 0.9915 \\
$A_{2}$ & 0.9879 & 0.9899 & 0.9843 & 0.9984 & 0.9853 \\
$A_{3}$ & 0.9858 & 0.9882 & 0.9881 & 0.9864 & 0.9893 \\
$A_{4}$ & 0.9935 & 0.9951 & 0.9889 & 0.9910 & 0.9896
\end{tabular}

Table 20

Similarity measure of Applicants vs. Positions using (7) and Type-3 score function

\begin{tabular}{cccccc}
\hline Applicants & $P_{1}$ & $P_{2}$ & $P_{3}$ & $P_{4}$ & $P_{5}$ \\
\hline$A_{1}$ & 0.9898 & 0.9920 & 0.9930 & 0.9915 & 0.9937 \\
$A_{2}$ & 0.9905 & 0.9918 & 0.9875 & 0.9986 & 0.9882 \\
$A_{3}$ & 0.9889 & 0.9911 & 0.9907 & 0.9892 & 0.9918 \\
$A_{4}$ & 0.9946 & 0.9958 & 0.9911 & 0.9930 & 0.9914
\end{tabular}

Table 21

Similarity measure of Applicants vs. Positions using (8) and Type-1 score function

\begin{tabular}{cccccc} 
Applicants & $P_{1}$ & $P_{2}$ & $P_{3}$ & $P_{4}$ & $P_{5}$ \\
\hline$A_{1}$ & 0.9745 & 0.9745 & 0.9885 & 0.9861 & 0.9888 \\
$A_{2}$ & 0.9697 & 0.9696 & 0.9772 & 0.9882 & 0.9882 \\
$A_{3}$ & 0.9863 & 0.9861 & 0.9745 & 0.9860 & 0.9855 \\
$A_{4}$ & 0.9817 & 0.9816 & 0.9772 & 0.9887 & 0.9855
\end{tabular}

Table 22

Similarity measure of Applicants vs. Positions using (8) and Type-2 score function 


\begin{tabular}{cccccc} 
Applicants & $P_{1}$ & $P_{2}$ & $P_{3}$ & $P_{4}$ & $P_{5}$ \\
\hline$A_{1}$ & 0.9595 & 0.9595 & 0.9802 & 0.9742 & 0.9803 \\
$A_{2}$ & 0.9515 & 0.9515 & 0.9655 & 0.9775 & 0.9794 \\
$A_{3}$ & 0.9744 & 0.9742 & 0.9595 & 0.9740 & 0.9733 \\
$A_{4}$ & 0.9733 & 0.9742 & 0.9655 & 0.9800 & 0.9733
\end{tabular}

Table 23

Similarity measure of Applicants vs. Positions using (8) and Type-3 score function

\begin{tabular}{cccccc} 
Applicants & $P_{1}$ & $P_{2}$ & $P_{3}$ & $P_{4}$ & $P_{5}$ \\
\hline$A_{1}$ & 0.9695 & 0.9694 & 0.9852 & 0.9807 & 0.9852 \\
$A_{2}$ & 0.9606 & 0.9605 & 0.9740 & 0.9802 & 0.9839 \\
$A_{3}$ & 0.9810 & 0.9807 & 0.9694 & 0.9803 & 0.9794 \\
$A_{4}$ & 0.9794 & 0.9807 & 0.9740 & 0.9849 & 0.9794
\end{tabular}

Table 24

Cosine Similarity measure of Applicants vs. Positions using (9) and Type-1 score function

\begin{tabular}{cccccc} 
Applicants & $P_{1}$ & $P_{2}$ & $P_{3}$ & $P_{4}$ & $P_{5}$ \\
\hline$A_{1}$ & 0.9806 & 0.9828 & 0.9930 & 0.9952 & 0.9954 \\
$A_{2}$ & 0.9790 & 0.9812 & 0.9822 & 0.9958 & 0.9934 \\
$A_{3}$ & 0.9830 & 0.9906 & 0.9770 & 0.9902 & 0.9884 \\
$A_{4}$ & 0.9880 & 0.9910 & 0.9842 & 0.9952 & 0.9944
\end{tabular}

Table 25

Cosine Similarity measure of Applicants vs. Positions using (9) and Type-2 score function

\begin{tabular}{cccccc} 
Applicants & $P_{1}$ & $P_{2}$ & $P_{3}$ & $P_{4}$ & $P_{5}$ \\
\hline$A_{1}$ & 0.9646 & 0.9690 & 0.9882 & 0.9886 & 0.9906 \\
$A_{2}$ & 0.9596 & 0.9642 & 0.9680 & 0.9894 & 0.9860 \\
$A_{3}$ & 0.9768 & 0.9812 & 0.9562 & 0.9778 & 0.9738 \\
$A_{4}$ & 0.9794 & 0.9856 & 0.9712 & 0.9906 & 0.9862
\end{tabular}

Table 26

Cosine Similarity measure of Applicants vs. Positions using (9) and Type-3 score function

\begin{tabular}{cccccc} 
Applicants & $P_{1}$ & $P_{2}$ & $P_{3}$ & $P_{4}$ & $P_{5}$ \\
\hline$A_{1}$ & 0.9770 & 0.9800 & 0.9924 & 0.9930 & 0.9942 \\
$A_{2}$ & 0.7728 & 0.9756 & 0.9790 & 0.9930 & 0.9910 \\
$A_{3}$ & 0.9854 & 0.9882 & 0.9718 & 0.9862 & 0.9836 \\
$A_{4}$ & 0.9864 & 0.9904 & 0.9812 & 0.9940 & 0.9914
\end{tabular}

All the horizontal decisions (From Table 4 to 26) have been presented in Table 27. Now, we have discussed horizontal decision. For instance, if we use (9) as a similarity measures then $A_{1}$ is appropriate for $P_{5}, A_{2}$ is appropriate for $P_{4}, A_{3}$ is appropriate for $P_{2}$ and $A_{4}$ is appropriate for $P_{4}$. Here it is seen that all $A_{2}$ is more appropriate than $A_{4}$. The same result occurs for Type 1, Type 2 and Type 3 score functions (see last three row of Table 27). Consequently, the obtained result is tallied with the result reported by Ejegwa (Ejegwa, 2019). So, we claimed that our similarity measure and score functions proposed here are justified. Similar conclusion may be drawn from other rows of table 27. All the vertical decisions (From Table 6 to 26) have been presented in Table 28.

Table 27

Horizontal Decisions taken from Table 6 to 26 


\begin{tabular}{|c|c|c|c|c|}
\hline Table 6 & $P_{5}$ & $P_{4}$ & $P_{2}$ & $P_{4}$ \\
\hline Table 7 & $P_{4}$ & $P_{4}$ & $P_{1}$ & $P_{4}$ \\
\hline Table 8 & $P_{4}$ & $P_{4}$ & $P_{4}$ & $P_{4}$ \\
\hline Table 9 & $P_{5}$ & $P_{4}$ & $P_{2}$ & $P_{4}$ \\
\hline Table 10 & $P_{5}$ & $P_{5}$ & $P_{3}$ & $P_{4}$ \\
\hline Table 11 & $P_{5}$ & $P_{5}$ & $P_{2}$ & $P_{4}$ \\
\hline Table 12 & $P_{4}$ & $P_{4}$ & $P_{4}$ & $P_{4}$ \\
\hline Table 13 & $P_{4}$ & $P_{4}$ & $P_{2}$ & $P_{4}$ \\
\hline Table 14 & $P_{4}$ & $P_{4}$ & $P_{2}$ & $P_{4}$ \\
\hline Table 15 & $P_{4}$ & $P_{4}$ & $P_{4}$ & $P_{5}$ \\
\hline Table 16 & $P_{4}$ & $P_{4}$ & $P_{4}$ & $P_{5}$ \\
\hline Table 17 & $P_{4}$ & $P_{4}$ & $P_{4}$ & $P_{4}$ \\
\hline Table 18 & $P_{5}$ & $P_{4}$ & $P_{5}$ & $P_{2}$ \\
\hline Table 19 & $P_{5}$ & $P_{4}$ & $P_{5}$ & $P_{2}$ \\
\hline Table 20 & $P_{5}$ & $P_{4}$ & $P_{5}$ & $P_{2}$ \\
\hline Table 21 & $P_{5}$ & $P_{4} / P_{5}$ & $P_{1}$ & $P_{4}$ \\
\hline Table 22 & $P_{5}$ & $P_{5}$ & $P_{1}$ & $P_{4}$ \\
\hline Table 23 & $P_{3} / P_{5}$ & $P_{5}$ & $P_{1}$ & $P_{4}$ \\
\hline Table 24 & $P_{5}$ & $P_{4}$ & $P_{2}$ & $P_{4}$ \\
\hline Table 25 & $P_{5}$ & $P_{4}$ & $P_{2}$ & $P_{4}$ \\
\hline Table 26 & $P_{5}$ & $P_{4}$ & $P_{2}$ & $P_{4}$ \\
\hline
\end{tabular}

Now, we have discussed vertical decision. For instance, if we use (9) as a similarity measures then positions $P_{1}, P_{2}$ and $P_{4}$ are appropriate for $A_{4}$, positions $P_{3}$ and $P_{5}$ are appropriate for $A_{1}$. The same result occurs for Type 1, Type 2 and Type 3 score functions (see last three row of Table 28). Consequently, the obtained result is tallied with the result reported by Ejegwa (Ejegwa, 2019). So, we claimed that our similarity measure and score functions proposed here are justified. Similar conclusion may be drawn from other rows of Table 28.

\section{Table 28}

Vertical Decisions taken from Table 6 to 26

$$
\begin{array}{lllll}
P_{1} & P_{2} & P_{3} & P_{4} & P_{5}
\end{array}
$$

\begin{tabular}{cccccc} 
Table No. & Appropriate for & Appropriate for & Appropriate for & Appropriate for & Appropriate for \\
\hline Table 6 & $A_{4}$ & $A_{4}$ & $A_{1}$ & $A_{4}$ & $A_{1}$ \\
Table 7 & $A_{4}$ & $A_{4}$ & $A_{1}$ & $A_{1}$ & $A_{1}$ \\
Table 8 & $A_{4}$ & $A_{4}$ & $A_{1}$ & $A_{2}$ & $A_{1}$ \\
Table 9 & $A_{3}$ & $A_{4}$ & $A_{1}$ & $A_{4}$ & $A_{1}$ \\
Table 10 & $A_{3}$ & $A_{4}$ & $A_{1}$ & $A_{4}$ & $A_{1}$ \\
Table 11 & $A_{3}$ & $A_{4}$ & $A_{1}$ & $A_{4}$ & $A_{1}$ \\
Table 12 & $A_{4}$ & $A_{4}$ & $A_{1}$ & $A_{1}$ & $A_{1}$ \\
Table 13 & $A_{4}$ & $A_{4}$ & $A_{1}$ & $A_{1}$ & $A_{1}$ \\
Table 14 & $A_{4}$ & $A_{4}$ & $A_{1}$ & $A_{1}$ & $A_{1}$ \\
Table 15 & $A_{4}$ & $A_{4}$ & $A_{1}$ & $A_{1}$ & $A_{1}$ \\
Table 16 & $A_{4}$ & $A_{4}$ & $A_{1}$ & $A_{1} / A_{2}$ & $A_{1} / A_{4}$ \\
Table 17 & $A_{4}$ & $A_{4}$ & $A_{1}$ & $A_{1}$ & $A_{1}$ \\
Table 18 & $A_{4}$ & $A_{4}$ & $A_{1}$ & $A_{2}$ & $A_{1}$ \\
\hline & & & & &
\end{tabular}

Table 28

Vertical Decisions taken from Table 6 to 26 (Continued) 
$\begin{array}{lllll}P_{1} & P_{2} & P_{3} & P_{4} & P_{5}\end{array}$

Table No. Appropriate for Appropriate for Appropriate for Appropriate for Appropriate for

\begin{tabular}{|cccccc}
\hline Table 19 & $A_{4}$ & $A_{4}$ & $A_{1}$ & $A_{2}$ & $A_{1}$ \\
\hline Table 20 & $A_{4}$ & $A_{4}$ & $A_{1}$ & $A_{2}$ & $A_{1}$ \\
\hline Table 21 & $A_{3}$ & $A_{3}$ & $A_{1}$ & $A_{4}$ & $A_{1}$ \\
\hline Table 22 & $A_{3}$ & $A_{3} / A_{4}$ & $A_{1}$ & $A_{4}$ & $A_{1}$ \\
\hline Table 23 & $A_{3}$ & $A_{3} / A_{4}$ & $A_{1}$ & $A_{4}$ & $A_{1}$ \\
\hline Table 24 & $A_{4}$ & $A_{4}$ & $A_{1}$ & $A_{2}$ & $A_{1}$ \\
\hline Table 25 & $A_{4}$ & $A_{4}$ & $A_{1}$ & $A_{4}$ & $A_{1}$ \\
Table 26 & $A_{4}$ & $A_{4}$ & $A_{1}$ & $A_{4}$ & $A_{1}$
\end{tabular}

\section{Concluding remarks}

The notion of Fermatean fuzzy sets is a new type of fuzzy sets to handle uncertain information more easily and effectively than the others existing fuzzy sets. Application of other fuzzy sets viz. intuitionistic fuzzy sets, Pythagorean fuzzy sets have been seen hugely in the existing literature but application of Fermatean fuzzy sets is very limited. So inspired from this, in this paper, we have solved Fermatean fuzzy multiple attribute group decision making problems where all the attributes are expressed in terms of Fermatean fuzzy sets. Firstly, we discuss the concept of Fermatean fuzzy sets. After that we propose some newly score function for ranking of Fermatean fuzzy sets. Then, first time we have proposed some score based similarity measures between two Fermatean fuzzy sets. We have also proposed score based cosine similarity measure between two Fermatean fuzzy sets. Applying all these similarity measures we have solved personnel appointments problem. Finally, a numerical example has been considered and solved for illustration purpose. The results obtained after applying our proposed score functions as well as different similarity measures coincide with the other which is available in existing literature. Therefore, it has been concluded that the proposed score functions of FFSs and different similarity measures proposed here can be used to solve pattern recognition problems, medical diagnosis problems and MCDM problems considering Fermatean fuzzy sets. In future research, one may apply the proposed similarity measures in the field of group decision making problems arising in the field of science, engineering and management science etc.

\section{References}

Atanassov, K. T. (1983). A Second type of Intuitionistic fuzzy sets. BUSE-FAL, 56, 66-70.

Atanassov, K. T. (1986). Intuitionistic fuzzy sets, Fuzzy Sets and Systems. 20, 87-96.

Atanassov, K. T. (2012). On Intuitionistic fuzzy sets theory. Springer, Berlin.

Atanassov, K. T. (2016). Geometric interpretation of the elements of the intuitionistic fuzzy objects. International Journal of Bioautomation, 20(S1), S27-S42.

Atanassov, K. T., \& Gargov, G. (1987). Interval valued intuitionistic fuzzy sets. Fuzzy Sets and Systems, 31, 1-17.

Atanassov, K. T., Vassilev, P.M., \& Tsvetkov, R. T. (2013). Intuitionistic fuzzy sets, measures and integrals, Professor Marin Drinov Academic publishing House, Sofia.

Bai, Z.Y. (2013). An interval valued intuitionistic fuzzy TOPSIS method based on an improved score function. The Scientific World Journal, 2013, 1-6.

Bellman, R.E., \& Zadeh, L.A. (1970). Decision making in a fuzzy environment. Management Science, 17, $141-164$.

Boltruk, E. (2018). Pythagorean fuzzy CODAS and its application to supplier selection in a manufacturing firm. Journal of Enterprise Information Management, 31, 550-564.

Chen, S. M. (1995). Measures of similarity between vague sets. Fuzzy Sets and Systems, 74(2), 217-223.

Chen, T-Y (2018). An outranking approach using a risk attitudinal assignment model involving Pythagorean fuzzy information and its application to financial decision making. Applied Soft Computing, 71, 460-487.

Ejegwa, P. A. (2019). Personnel Appointments: A Pythagorean fuzzy sets approach using similarity measure. Journal of information and Computing Science, 14(2), 094-102.

Ejegwa, P. A. (2020), Distance and similarity measures of Pythagorean fuzzy sets, Granular Computing, 5, $225-238$.

Fei, L.,Wang, H., Chen, L., \& Deng, Y., A new vector valued similarity measure for intuitionistic fuzzy sets based on OWA operators. Iranian Journal of Fuzzy Systems, 15(5), 31-49.

Garg, H. (2017). Anew improved score function of an interval valued Pythagorean fuzzy set based topsis method. International Journal of Uncertainty Quantification, 7, 463-474.

Garg, H. (2018). A linear programming method based on an improved score function for interval valued Pythagorean fuzzy numbers and its application to decision-making. International Journal of Uncertainty Fuzziness Knowledge Based System, 26, 67-80.

Geng, Y., Liu, P., Teng, F., \& Liu, Z. (2017). Pythagorean fuzzy uncertain linguistic TODIM method and their application to multiple criteria group decision making. International Journal of Intelligent Systems, 33, 3383-3395. 
Gorzalczany, M. B. (1987). A method of inference in approximate reasoning based on interval-valued fuzzy sets. Fuzzy Sets and Systems, 21(1), 1-17.

Gou, X., Xu, Z., \& Liao, H.(2016). Alternative queuing method for multiple criteria decision making with hybrid fuzzy and ranking information. Information Science, 357, 144-160.

Hung, W. L., \& Yang, M. S. (2004). Similarity measures of intuitionistic fuzzy sets based on Hausdorff distance. Pattern Recognition Letters, 25(14), 1603-1611.

Jing, N., Xian, S., \& Xiao, Y. (2017). Pythagorean triangular fuzzy linguistic bonferroni mean operators and their application for multi-attribute decision making, In: $2^{\text {nd }}$ IEEE international conference on computational intelligence and applications (ICCIA), 435-439.

Li, D., \& Cheng, C.(2002). New similarity measures of intuitionistic fuzzy sets and application to pattern recognitions. Pattern Recognition Letters, 23(1-3), 221-225.

Li, Z., Wei, G.,\& Lu, M.(2018). Pythagorean fuzzy Hamy mean operators in multiple attribute group decision making and their application to supplier selection. Symmetry, 10, 505.

Liang, Z., \& Shi, P. (2003). Similarity measures on intuitionistic fuzzy sets. Pattern Recognition Letters, 24(15), 26872693.

Lin, Y-L, Ho, L-H, Yeh, S-L, \& Chen, T-Y ( 2018). A Pythagorean fuzzy topsis method based on novel correlation measures and its application to multiple criteria decision analysis of inpatient stoke rehabilitiation. International Journal of Computer Intelligence System, 12(1), 410-425.

Liu, D., Chen, X., \& Peng, D.(2018). Cosine Similarity Measure between Hybrid Intuitionistic Fuzzy Sets and Its Application in Medical Diagnosis. Computational and Mathematical Methods in Medicine, 2018, Article ID 3146873, 7 pages.

Liu, D., Chen, X.,\& Peng, D.(2018). The intuitionistic fuzzy linguistic cosine similarity measure and its application in pattern recognition. Complexity, 2018, Article ID 9073597, 11 pages.

Liu, D., Liu, Y., \& Wang L.(2019). Distance measure for Fermatean fuzzy linguistic term sets based on linguistic scale function: An illustration to the TODIM and TOPSIS methods. International Journal of Intelligent Systems, 34(11), 2807-2834.

Nguyen, H. (2016). A novel similarity/dissimilarity measure for intuitionistic fuzzy sets and its application in pattern recognition. Expert Systems with Applications, 45, 97-107.

Pappis, C. P., \& Karacapilidis, N. I. (1993). A comparative assessment of measures of similarity of fuzzy values. Fuzzy Sets and Systems, 56(2), 171-174.

Qin, J. (2018). Generalized Pythagorean fuzzy Maclaurin symmetric means and its application to multiple attribute sir group decision model. International Journal of Fuzzy System, 20, 943-957.

Senapati, T., \& Yager, R. R. (2019). Fermatean fuzzy weighted averaging/geometric operators and its application in multicriteria decision-making methods. Engineering Applications of Artificial Intelligence, 85, 112-121.

Senapati, T., \& Yager, R. R. (2020). Fermatean fuzzy sets. Journal of Ambient Intelligence and Humanized Computing, 11, 663-674.

Turksen, I.B. (1986). Interval valued fuzzy sets based on normal forms, Fuzzy Sets and Systems, 20(2), 191-210.

Wan, S-P, Li, S-Q, \& Dong, J-Y (2018). A three phase method for Pythagorean fuzzy multi attribute group decision making and application to haze management. Computers and Industrial Engineering, 123, 348-363.

Yager, R. R. (2013). Pythagorean fuzzy subsets, In: 2013 joint IFSA world congress and NAFIPS annual meeting (IFSA/ NAFIPS), 57-61.

Yager, R. R. (2014). Pythagorean membership grades in multi-criteria decision making, IEEE Transactions on Fuzzy Systems, 22, 958-965.

Ye, J. (2009). Multicriteria fuzzy-decision making method based on a novel accuracy function under interval valued intuitionistic fuzzy environment. Expert Systems with Applications, 36(3), 6899-6902.

Ye, J. (2011). Cosine similarity measures for intuitionistic fuzzy sets and their applications. Mathematical and Computer Modeling, 53(1-2), 91-97.

Ye, J. (2013). Interval-valued intuitionistic fuzzy cosine similarity measures for multiple attribute decision-making, International Journal of General Systems, 42(8), 883-891.

Zadeh, L. A. (1965). Fuzzy Sets, Information and Control, 8, 338-353.

Zhang, R., Ashuri, B., \& Deng, Y. (2018). A novel method for forecasting time series based on fuzzy logic and visibility graph. Advances in Data Analysis and Classification, 11(4), 759-783.

Zhang, X, \& Xu, Z. (2014). Extention of TOPSIS to multiple criteria decision making with Pythagorean fuzzy sets. International Journal of Intelligent System, 29, 1061-1078.

Zhang, X.(2016). A novel approach based on similarity measure for Pythagorean fuzzy multiple criteria group decision making. International Journal of Intelligent System, 31, 593-611.

Zhang, Z., Yang, J., Ye, Y., Hu, Y., \& Zhang, Q. (2012). A type of score function on intuitionistic fuzzy sets with double parameters and its application to pattern recognition and medical diagnosis. Procedia Engineering, $29,4336-4342$.

Zhou, J., Su, W., Balezentis, T., \& Streimikiene, D. (2018). Multiple criteria group decision making considering symmetry with regards to the positive and negative ideal solution via the Pythagorean normal cloud model for application to economic decision. Symmetry, 10(5), 140. 
Zhou, L., Z. Tao, H. Chen, \& Liu, J. (2014). Intuitionistic fuzzy ordered weighted cosine similarity measure. Group Decision and Negotiation, 23(4), 879-900.

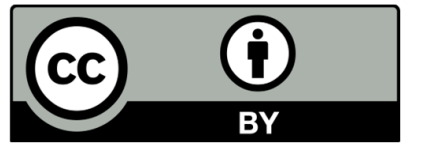

(C) 2022 by the authors; licensee Growing Science, Canada. This is an open access article distributed under the terms and conditions of the Creative Commons Attribution (CC-BY) license (http://creativecommons.org/licenses/by/4.0/). 